\title{
RS485 Image Sensor for Digital Cinema System
}

\author{
Eunju Kim, ${ }^{1}$ Seokhoon Kang, ${ }^{2}$ and Sangsoon Lee $^{3}$ \\ ${ }^{1}$ High-Tech S/W Research Center, Incheon National University, 119 Academy-ro, Yeonsu-gu, Incheon 406-772, Republic of Korea \\ ${ }^{2}$ Department of Embedded Systems Engineering, Incheon National University, 119 Academy-ro, Yeonsu-gu, \\ Incheon 406-772, Republic of Korea \\ ${ }^{3}$ Department of Computer Engineering, Gachon University, 1342 Seongnam-daero, Sujeong-gu, Seongnam-si, \\ Kyunggi-do, Seongnam 461-701, Republic of Korea \\ Correspondence should be addressed to Sangsoon Lee; sslee@gachon.ac.kr
}

Received 21 January 2015; Accepted 19 March 2015

Academic Editor: Wei Wu

Copyright (C) 2016 Eunju Kim et al. This is an open access article distributed under the Creative Commons Attribution License, which permits unrestricted use, distribution, and reproduction in any medium, provided the original work is properly cited.

\begin{abstract}
To activate various devices using RS485, a repeater is generally used. In current digital cinema systems, each device is controlled with RS485 by mixing RS485 and DMX512. However, as today's cinema equips hundreds of 4D chairs and the environmental directors, it is nearly infeasible for the legacy system to control. To this end, this paper designs and implements a new system which makes hundreds of 4D chairs and the environmental directors be controlled simultaneously exploiting RS485 network topology and its repeaters. The proposed approach is tested in a real-time system for assessing the performance by Paessler Router Traffic Grapher (PRTG) in Windows environment. Simulation results show that the tested system supports 4D chairs and their motions are well operated simultaneously with RS485.
\end{abstract}

\section{Introduction}

A digital cinema indicates the usage of digital technology to deliver and present motion pictures in contrast to the historical usage of motion picture film [1]. Movies can be delivered via internet and hard drives or devoted to web or satellite links or optical disks such as blu-ray disc [2]. Digital movies are presented adopting a digital projector instead of a traditional film projector. Thus, digital cinema is different from HDTV (high-definition television) and does not rely on adopting SD or HDTV standards [3]. In general, resolutions of digital cinema are represented by the horizontal pixel count, usually $2048 \times 1080$ (or 2.2 megapixels) to $4096 \times$ 2160 (or 8.8 megapixels). As digital cinema technique was enhanced in early $2010 \mathrm{~s}$, most of the theaters across the world have transferred to digital [4].

Four dimensional (4D) film is a term of marketing for an entertainment presentation system which is advanced form of three dimensional (3D) film with physical possessions that transpire in the theatre in operation with the film [5]. The accomplishments conducted in a 4D film may include lamp, wind, rain, and vibration. The seats in $4 \mathrm{D}$ place may oscillate or move a few inches during the movie [6]. Some other effects contain water sprays, air jets, and leg and back ticklers. In addition, hall effects may include rain, smoke, lightning, and air bubbles, and scent can be considered for $4 \mathrm{D}$ effects. Since the physical effects are expensive to realize, 4D films are most often accomplished in custom-built theatres such as amusement or theme parks. Some examples of $4 \mathrm{D}$ films are "Journey to the Center of the Earth" and "Avatar," which were presented at movie theatres with $4 \mathrm{D}$ versions [7].

Recently, research on IT convergence techniques for film production and film screening are widely studied. Analogtype theater is changing to digital-type theater, and interests in digital-type theater increase for 4D-type theater which yields reality that $3 \mathrm{D}$ movies did not provide [8]. A 3D theater is giving a three-dimensional effect to viewers with special glasses such as polarized glasses [9]. In addition, a future-oriented $4 \mathrm{D}$ theater helps viewers to try special effects affecting five senses such as wind, vapor, chair vibration, scent, and special lighting $[10,11]$.

In a traditional $4 \mathrm{D}$ digital theater, an environmental system that generates device controller of lamping system and a device controller of sensing of reality are separated [12]. That 
TABLE 1: Environmental parameters.

\begin{tabular}{|c|c|c|c|c|c|c|c|c|}
\hline \multirow{3}{*}{ Equipment } & Starter & \multicolumn{3}{|c|}{ Delimiter } & \multicolumn{3}{|c|}{ Parameter } & \multirow[t]{2}{*}{ CRC } \\
\hline & & & & & 1 & 2 & 3 & \\
\hline & 1 (STX) & 2 & 3 & 4 & 5 & 6 & 7 & 12 \\
\hline \multicolumn{9}{|l|}{ LED lighting } \\
\hline Actual value & 0x02 & $0 \mathrm{x} 4 \mathrm{c}$ & $0 \times 45$ & $0 \times 44$ & \multicolumn{2}{|c|}{$0 \sim 60000$} & $0 \sim 255$ & $0 \sim 255$ \\
\hline Meaning & Begin & “L” & “E” & “D” & ID top & ID down & Type booking & $1 \sim 255$ data \\
\hline \multicolumn{9}{|l|}{ Fan } \\
\hline Actual value & $0 \times 02$ & $0 \times 46$ & $0 \times 41$ & $0 \mathrm{x} 4 \mathrm{E}$ & \multicolumn{2}{|c|}{$0 \sim 60000$} & $0 \sim 255$ & $0 \sim 255$ \\
\hline Meaning & Begin & "F" & "A" & "N" & ID top & ID down & Type booking & $1 \sim 255$ data \\
\hline \multicolumn{9}{|l|}{ Motion } \\
\hline Actual value & 0x02 & $0 \times 4 d$ & $0 \mathrm{x} 4 \mathrm{f}$ & $0 \times 54$ & \multicolumn{2}{|c|}{$0 \sim 60000$} & $0 \sim 255$ & $0 \sim 255$ \\
\hline Meaning & Begin & M & $\mathrm{O}$ & $\mathrm{T}$ & ID top & ID down & Type booking & $1 \sim 255$ data \\
\hline
\end{tabular}

is, DMX512 protocol is adopted to control lamping and RS45 image sensor is adopted to control motions, chair devices, and environments generating devices [13]. To this end, two types of controllers must be realized to control each device and generally they cause high power consumption and complex communication lines [14]. In addition, controlling all the subdevices precisely and simultaneously is hard to achieve [15-17]. Therefore, a stable device controller system is needed which can control all the subdevices precisely and provide autoinspection and calibration of real-time operation.

In this paper, RS485 image sensor based digital cinema system is proposed. The system is designed to integrate motion bases, chair devices, and other environmental rendering devices as well as lighting under RS485 image communication. This paper is organized as follows. Section 2 introduces $4 \mathrm{D}$ device control system. The proposed system is explained in Section 3. Section 4 provides simulation results and conclusion remarks are described in Section 5.

\section{Proposed Design}

2.1. Image Coding. The digital cinema architecture utilizes the JPEG standard to realize a real-time decoder. Due to the communication traffic and storage cost, interframe such as video coding can be better choice for movie compression. However, there are some reasons that we selected JPEG:

(1) There is no global standard for RGB compression with 30 bits or more.

(2) Intraframe coding methods remain significant due to their supporting of video editing.

(3) JPEG is easier standard for editing.

(4) JPEG decoder is more reliable in the error condition.

Therefore, we used JPEG for our digital cinema system.

2.2. Real-Time Decoder. The decoder is able to realize realtime decompression with higher speed of $3 \mathrm{G}$ pixels per minute using parallel approach. The decoder is comprised of two circuit blocks: PC/LINUX part with GbE interface and JPEG2000 decoder boards. Four boards are established on the PCI-X-bus for processing 24 frames of $8 \mathrm{M}$ pixels with 36bit RGB color images in a second. The color standards may be changed such as RGB, YCbCr, or HSV.
2.3. The 4D Device Control System. The 4D device control system is working for the environmental rendering of digital theater. To investigate 4D device control system, we tested some characteristics including chair vibration, wind, vapor, scent, special lighting, and spray in the hardware configuration. We also tested LED lighting, fan, and vibration among the various environmental rendering devices.

As RS485 image sensor only enables multiple connections of various masters and gives half duplex communication, two wires are exploited for sender and receiver. The ID is assigned to each device. If a master sends data " 1 " to master " $m$," all devices receive the data simultaneously. However, other devices except master " $m$ " ignore the data once they realize the data is not for them. Table 1 shows data configuration table of RS485 image sensor protocol.

Conventional studies performed simulations for LED lighting, fan, and vibration among the diverse environmental rendering device and displayed their accuracy in real-time data transmission. Although previous researches studied data transmission for RS485 image sensor, those studies did not fully provide comprehensive understanding of environmental rendering devices, motion, and chair devices. To alleviate this issue, we verify data transmission for RS485 image sensor and design digital cinema system which is able to provide all the environmental rendering devices, motion, and chair devices.

\section{Configuration of the Proposed Digital Cinema System}

Let us consider structure of conventional digital cinema system.

Step 1. Main PC supports connectivity between the Internet and sites based on Ethernet. Using 4D system interface shown in Figure 1, the entire system is controlled.

Step 2. This stage is for network server, which monitors devices. In addition, network server converts data between main PCs.

Step 3. This is the last step which belongs to physical layer devices. RS485 works for communication and is designed 


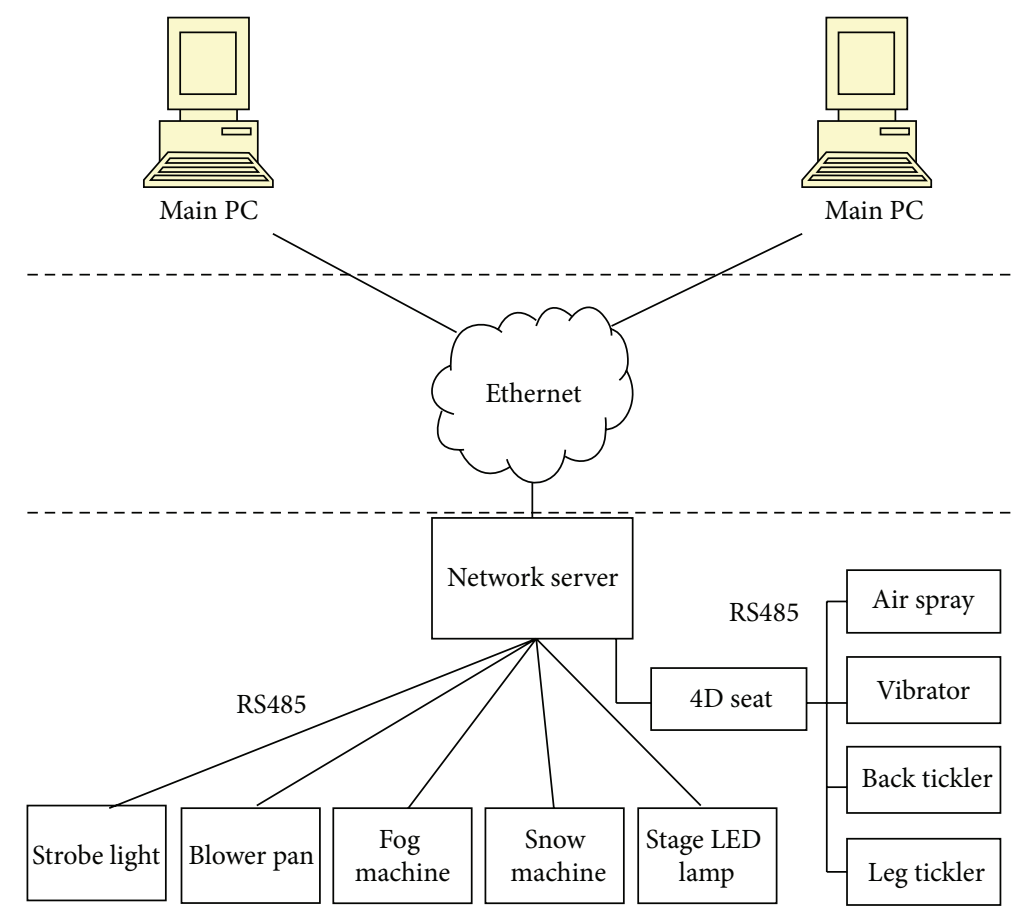

Figure 1: Conventional digital cinema system.

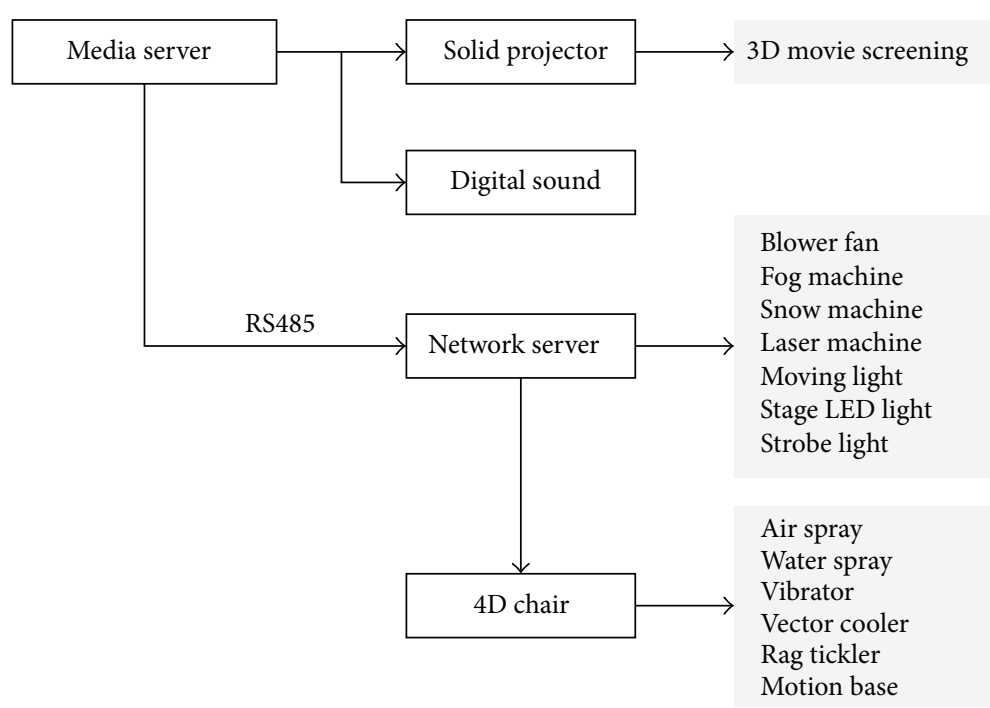

FIgURE 2: Configuration of digital cinema system.

to expand. However, cinema has hundreds of $4 \mathrm{D}$ chairs; therefore compatibility with existing system is a big issue. In our system, we use RS485 network bus topology and repeater to control hundreds of $4 \mathrm{D}$ chairs and environmental productions.

Figure 2 shows the proposed digital cinema system configuration. When a data file is entered in a media server, a driving controller delivers data to each device through RS485 image sensor. Each device provides environmental rendering effect.
3.1. Design of Digital Cinema System. The driving controller of digital cinema system activates environmental rendering devices in synchronization with screen display as well as devices which are installed on a chair such as motion base. The driving controller of digital cinema system can be installed in automatic manner through touch screen and yields tests for some operations.

3.2. Protocol Definition of RS485 Image Sensor. To define RS485 image sensor protocol, we take into account motion, chair device, and environment devices simultaneously. To 
TABLE 2: Mandatory instruction set for environmental devices.

\begin{tabular}{|c|c|c|c|c|}
\hline Equipment & & Delimiter & & Contents \\
\hline \multicolumn{5}{|l|}{ LED lighting } \\
\hline Actual value & $0 \mathrm{x} 4 \mathrm{c}$ & $0 \times 45$ & $0 \times 44$ & \multirow{2}{*}{ Color, brightness, and time adjustment of LED light } \\
\hline Meaning & “L” & "E” & "D” & \\
\hline \multicolumn{5}{|l|}{ Strobe lighting } \\
\hline Actual value & $0 \times 53$ & $0 \times 54$ & $0 \times 52$ & \multirow{2}{*}{$\begin{array}{l}\text { Brightness, blink rate, and holding time adjustment of } \\
\text { the strobe light }\end{array}$} \\
\hline Meaning & “S” & “T” & “R” & \\
\hline \multicolumn{5}{|l|}{ Heater } \\
\hline Actual value & $0 \times 48$ & $0 \times 45$ & $0 \times 54$ & \multirow{2}{*}{ Temperature of the heater } \\
\hline Meaning & “H” & “E” & “T” & \\
\hline \multicolumn{5}{|l|}{ Fan } \\
\hline Actual value & $0 \times 46$ & $0 \times 41$ & $0 \mathrm{x} 4 \mathrm{E}$ & \multirow{2}{*}{ Wind speed of the fan } \\
\hline Meaning & "F" & “A” & "N" & \\
\hline \multicolumn{5}{|l|}{ Fog } \\
\hline Actual value & $0 \times 46$ & $0 \mathrm{x} 4 \mathrm{~F}$ & $0 \times 47$ & \multirow{2}{*}{ Fogging degree of fog machine } \\
\hline Meaning & "F" & “O” & “G” & \\
\hline \multicolumn{5}{|l|}{ Curtain } \\
\hline Actual value & $0 \times 43$ & $0 \times 54$ & $0 \mathrm{x} 4 \mathrm{E}$ & \multirow{2}{*}{ Curtain's opening degree } \\
\hline Meaning & “C” & “T” & "N" & \\
\hline \multicolumn{5}{|l|}{ Flash } \\
\hline Actual value & $0 \times 46$ & $0 \times 4 C$ & $0 \times 53$ & \multirow{2}{*}{ Brightness and action adjustment of flash } \\
\hline Meaning & "F" & "L” & “S” & \\
\hline \multicolumn{5}{|c|}{ Ground LED light } \\
\hline Actual value & $0 \times 55$ & $0 \times 4 c$ & $0 \times 44$ & \multirow{2}{*}{ Color, brightness, and motion of ground LED lighting } \\
\hline Meaning & $\mathrm{U}$ & $\mathrm{L}$ & $\mathrm{D}$ & \\
\hline \multicolumn{5}{|c|}{ Ceiling LED light } \\
\hline Actual value & $0 \times 52$ & $0 \times 4 c$ & $0 \times 44$ & \multirow{2}{*}{ Color, brightness, and motion of ceiling LED lighting } \\
\hline Meaning & $\mathrm{R}$ & $\mathrm{L}$ & $\mathrm{D}$ & \\
\hline \multicolumn{5}{|l|}{ Moving light } \\
\hline Actual value & $0 \times 4 d$ & $0 \times 56$ & $0 \mathrm{x} 4 \mathrm{c}$ & \multirow{4}{*}{$\begin{array}{l}\text { Color of the moving light, operating time, blink rate, } \\
\text { movement speed, and position adjustment }\end{array}$} \\
\hline Meaning & "M" & "V" & "L" & \\
\hline Actual value & $0 \times 4 d$ & $0 \times 56$ & $0 \times 4 c$ & \\
\hline Meaning & "M" & "V" & “L” & \\
\hline
\end{tabular}

this end, we used separators for each two to four bytes to distinguish motion, chair device, and environment device commands. We also defined a protocol by taking into account the scalability of environment and chair devices. The basic function of each device is coded as a specific parameter in a command set. When a command is delivered to each device, it activates defined operation. The command is comprised of 12 bytes including 115,200 bps, 8 bit data, 1 stop bit, and 0 parity bit. Consider

$$
\text { STX + separators }(3 \text { byte })+\text { variable }(7 \text { byte })+\text { CR } .
$$

Based on (1), command sets are made as Table 2.

To connect hundreds of chairs and servers, we design RS485 repeater. This is to ensure the stability of the communication between the isolated signals and to be able to play the best communication state through the embedded MCU. The designed system may have total of 4 channels; 1 input signal of each channel is reoutput after isolating via the SN75176.

3.3. Signal Processing. A multiple controller is executed to allot multiple signal processing among various devices, fieldeffect transistor (FET) board control, power check control, and multipoint control unit (MCU) monitoring, to raise stability. The controller is divided into main MCU, motor MCU, and monitor MCU to achieve mutual monitoring and help. The main MCU is responsible for signal processing and examines encoded signal, digital input/output signals. In addition, the main MCU generates pulse width modulation (PWM) signal which delivers an appropriate driving signal to the FBT board.

The FET board is controlled by the motor MCU. It stops motion base and reports possible errors to the main MCU when an error alert is delivered. The monitor MCU oversees 


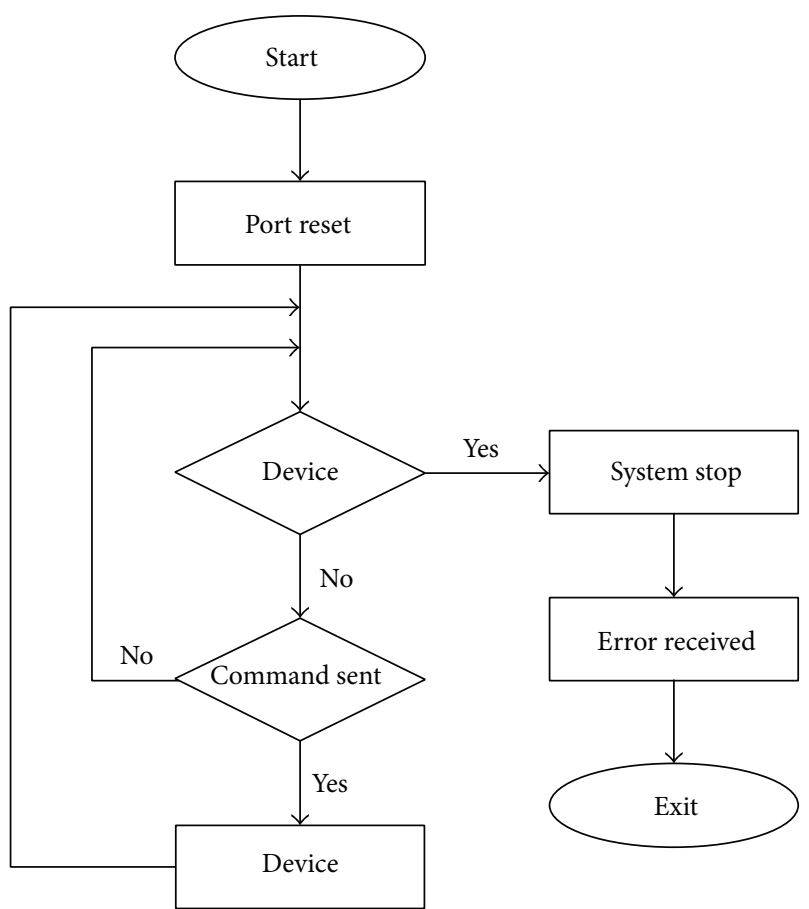

FIGURE 3: Block diagram of the motor and monitor for MCU operation.

power and monitors of other MCUs. The signals overseen by monitor MCU are significant to the safety of the entire system and can tune power supply to the system. The operation sequence of motor and monitor MCUs is shown in Figure 3.

Figure 4 shows the proposed digital cinema system. Step 1 is main PC which supports connectivity between the Internet and sites based on Ethernet. Step 2 is network server, which is able to monitor the devices. Step 3 is physical layer, which is comprised of devices, and commination between devices is RS485. In this paper, we propose a method with repeater which is free of problems on the network even when the number of $4 \mathrm{D}$ chairs increases. Figure 4 shows improved digital cinema system.

3.4. RS485 Repeater Design. Repeaters are used only when the network has the same structure, and they belong to physical layer. We designed RS485 repeater for multiple system communication. It can be connected to at least hundreds of $4 \mathrm{D}$ chair system and the server, which ensures the stability of the communication between insulated signals. The system has 4 channels; input signal of each channel is isolated via SN75176 and output again.

\section{Experimental Results}

Simulations of the improved digital cinema system must control hundreds of $4 \mathrm{D}$ chairs and a motion base to exactly and simultaneously operate through the RS485 image sensor. Therefore, our simulation was tested by installing 100 4D chairs under the corresponding motion base. The hardware environments are indicated in Table 3. The operating system
TABLE 3: Environments for simulation.

\begin{tabular}{|c|c|}
\hline Type & Hardware spec \\
\hline \multirow{14}{*}{ 4D chair } & (i) Seats: 2 persons \\
\hline & (ii) Woofer power: $\mathrm{DC} 24 \mathrm{~V}$ \\
\hline & (iii) Woofer quantity: $6 \mathrm{Ea}$ \\
\hline & (iv) Effect accessory: water spray 1 set \\
\hline & (v) Air spray 1 set \\
\hline & (vi) Vibrator 1 set \\
\hline & (vii) Leg tickler 1 set \\
\hline & (viii) Frame material: steel \\
\hline & (ix) Cover material: fabric \\
\hline & (x) Seats material: urethane \\
\hline & (xi) Accessory: cup holder \\
\hline & (xii) Side cover material: wood \\
\hline & (xiii) Weight: $40 \mathrm{Kg}$ \\
\hline & (xiv) Include wire, hose, and connector \\
\hline \multirow{8}{*}{$\begin{array}{l}4 \mathrm{D} \text { motion } \\
\text { base }\end{array}$} & (i) Dimension $(W \times D \times H)$ : $1,260 \times 670 \times 450$ \\
\hline & (ii) Power: $220 \mathrm{VAC} \pm 10 \%, 50 / 60 \mathrm{~Hz}$, Max. $1,000 \mathrm{~W}$ \\
\hline & (iii) Motion range: Pitch $=$ Max. 6 degrees \\
\hline & (iv) Roll = Max. 6 degrees \\
\hline & (v) Heave = Max. $44 \mathrm{~mm}$ \\
\hline & (vi) $3 \mathrm{DOF}$ (degrees of freedom) \\
\hline & (vii) Effect accessory: leg tickler 1 set \\
\hline & (viii) Weight: $50 \mathrm{Kg}$ \\
\hline
\end{tabular}

(OS) of the software environment was Windows 8 and the program language we used is $\mathrm{C}++$. The adopted network environment was Windows 8-based Paessler Router Traffic Grapher (PRTG) simulator.

In the previous works, $4 \mathrm{D}$ chair, motion, air injection, water spray, and vibration were considered as personal experience. However, in this study, we excluded group experience and only considered personal experience, that is, 4D chair and motion. Table 4 shows environmental device parameter sets for $4 \mathrm{D}$ chair and motion. The environments guided device parameter values are set in Table 4 and stored as a metadata image file. The main screen runs both image data on left and right to produce 3D effect simultaneously. The data transmission is divided by synchronous and asynchronous connections according to the time positions of the transmitting and receiving side. The synchronous data is transmitted in a noncharacter block unit such as frame. In other words, data is made by a predetermined number of strings between the transmitting side and the receiving side, with a format of packet at a time. The asynchronous transfer is motivated by sending information to noncharacter unit blocks. The synchronous transmission has character synchronization method, bit synchronization method, and the frame synchronization method. In this paper, we used character synchronous system which is the most reliable among all synchronous processing. To align characters we add STX in front of blocks and add ETX at the end of blocks to indicate beginning and the end of the transmission data. 
TABLE 4: Device parameter values for environments.

\begin{tabular}{|c|c|c|c|c|c|c|c|c|c|c|c|c|}
\hline \multirow{2}{*}{ Equipment } & \multirow{2}{*}{$\begin{array}{c}\text { Starter } \\
1 \text { (STX) }\end{array}$} & \multicolumn{3}{|c|}{ Delimiter } & \multicolumn{2}{|c|}{ Parameter 1 Parameter 2} & \multirow{2}{*}{$\begin{array}{c}\text { Parameter } 3 \\
7 \\
\end{array}$} & \multirow{2}{*}{$\begin{array}{c}\text { Parameter } 4 \\
8 \\
\end{array}$} & \multirow{2}{*}{$\begin{array}{c}\text { Parameter } 5 \\
9\end{array}$} & \multirow{2}{*}{$\begin{array}{c}\text { Parameter } 6 \\
10 \\
\end{array}$} & \multirow{2}{*}{$\begin{array}{c}\text { Parameter } 7 \\
11 \\
\end{array}$} & \multirow{2}{*}{$\begin{array}{c}\text { CRC } \\
12 \\
\end{array}$} \\
\hline & & 2 & 3 & 4 & 5 & 6 & & & & & & \\
\hline \multicolumn{13}{|l|}{ Notion } \\
\hline Actual value & e $0 \mathrm{x} 02$ & $0 \times 4 d$ & $0 \times 4 f$ & $0 \times 54$ & & 80000 & $\mathrm{XX}$ & $\mathrm{XX}$ & $\mathrm{XX}$ & $\mathrm{XX}$ & $\mathrm{XX}$ & $0-255$ \\
\hline Meaning & Begin & M & $\mathrm{O}$ & $\mathrm{T}$ & ID & ID & Type & Type & Type & Type & Type & Data \\
\hline \multicolumn{13}{|l|}{ 4D chair } \\
\hline Actual value & e $0 \times 02$ & $0 \times 53$ & $0 \times 4 f$ & $0 \times 4 c$ & & 80000 & $\mathrm{XX}$ & $\mathrm{XX}$ & $\mathrm{XX}$ & $\mathrm{XX}$ & $\mathrm{XX}$ & $0-255$ \\
\hline Meaning & Begin & S & $\mathrm{O}$ & $\mathrm{L}$ & ID & ID & Type & Type & Type & Type & Type & Data \\
\hline
\end{tabular}

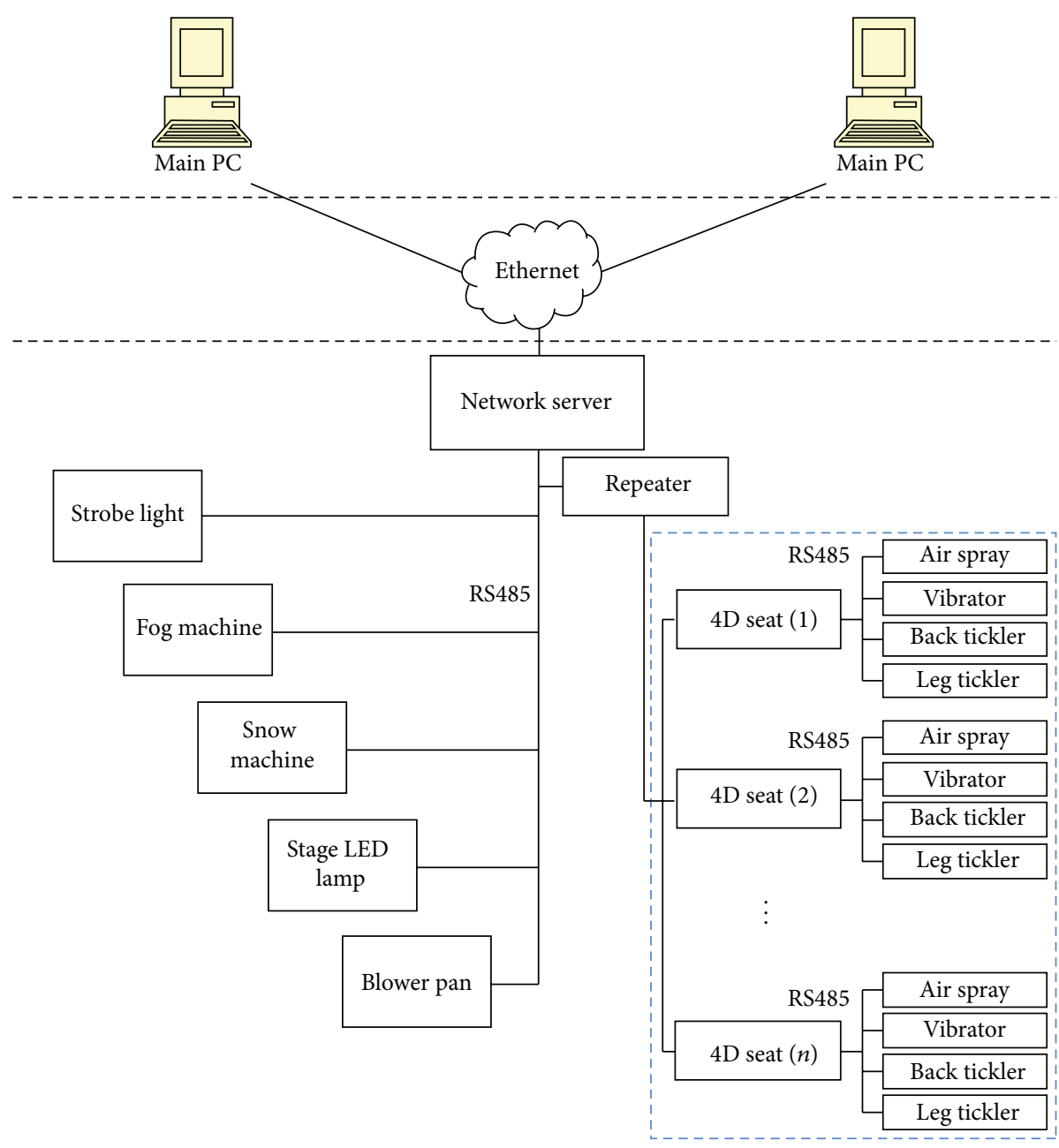

Figure 4: Proposed digital cinema system.

The motion chairs and environmental production device parameter values that were set in Table 4 are shown in MOT and SOT. The reason our system starts with STX is that we adopted character synchronous system for its stability in high-speed processing. To inform the beginning and the end, the character synchronous system adds STX to the front and ETX to the end of transmitted data.

From the main screen it can be found that 15 Gbyte movie file is well transmitted to receiver through the $100 \mathrm{Mbps}$ interface of repeater. This confirms that 4D motion and chair were correctly operated when server and each device node information is properly transmitted without loss. Figure 5 shows transition of motor torques and motor power for M1, M2, and M3. The M1 motor power was $280 \mathrm{~W}$. Based on the simulation, we designed and produced motion base structure. Figure 6 shows a simulation for selected 3DOF motion base dimension.

\section{Conclusions}

The most representative tangible personal experience factors of $4 \mathrm{D}$ theaters are $4 \mathrm{D}$ chair and $4 \mathrm{D}$ cinema, and hands-on 


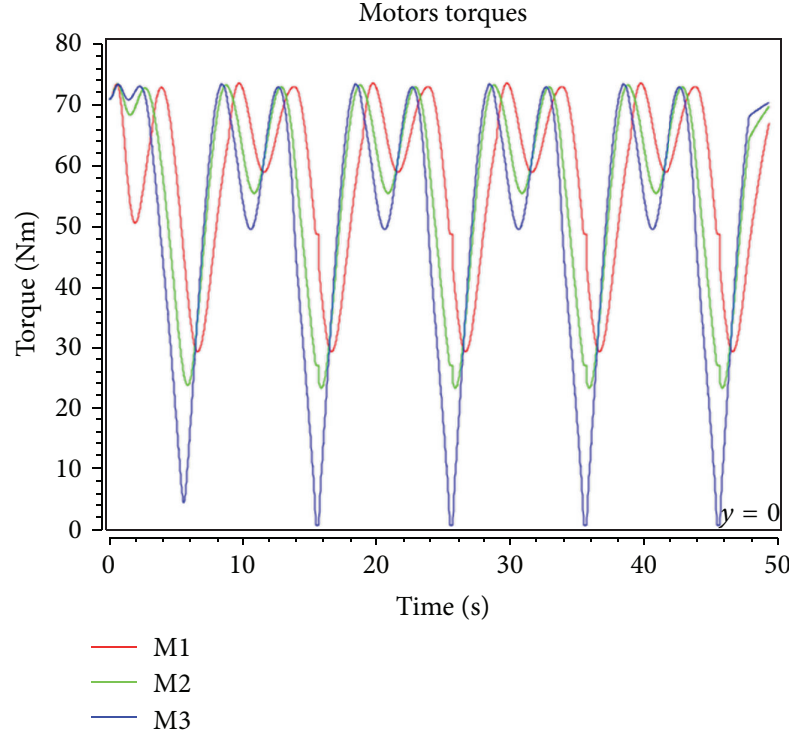

(a)

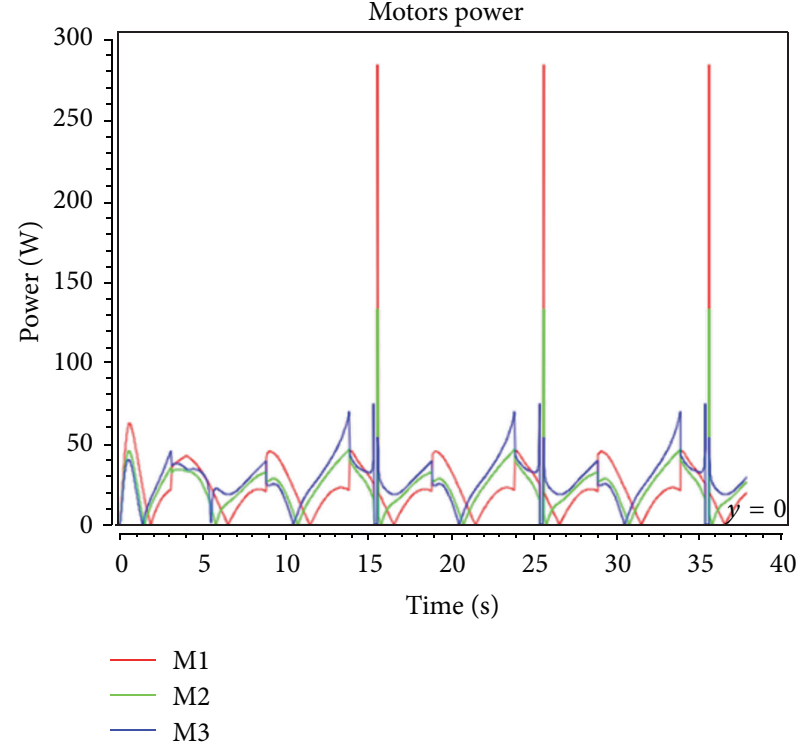

(b)

Figure 5: (a) Transition of motor torques for M1, M2, and M3. (b) Transition of motor power for M1, M2, and M3.
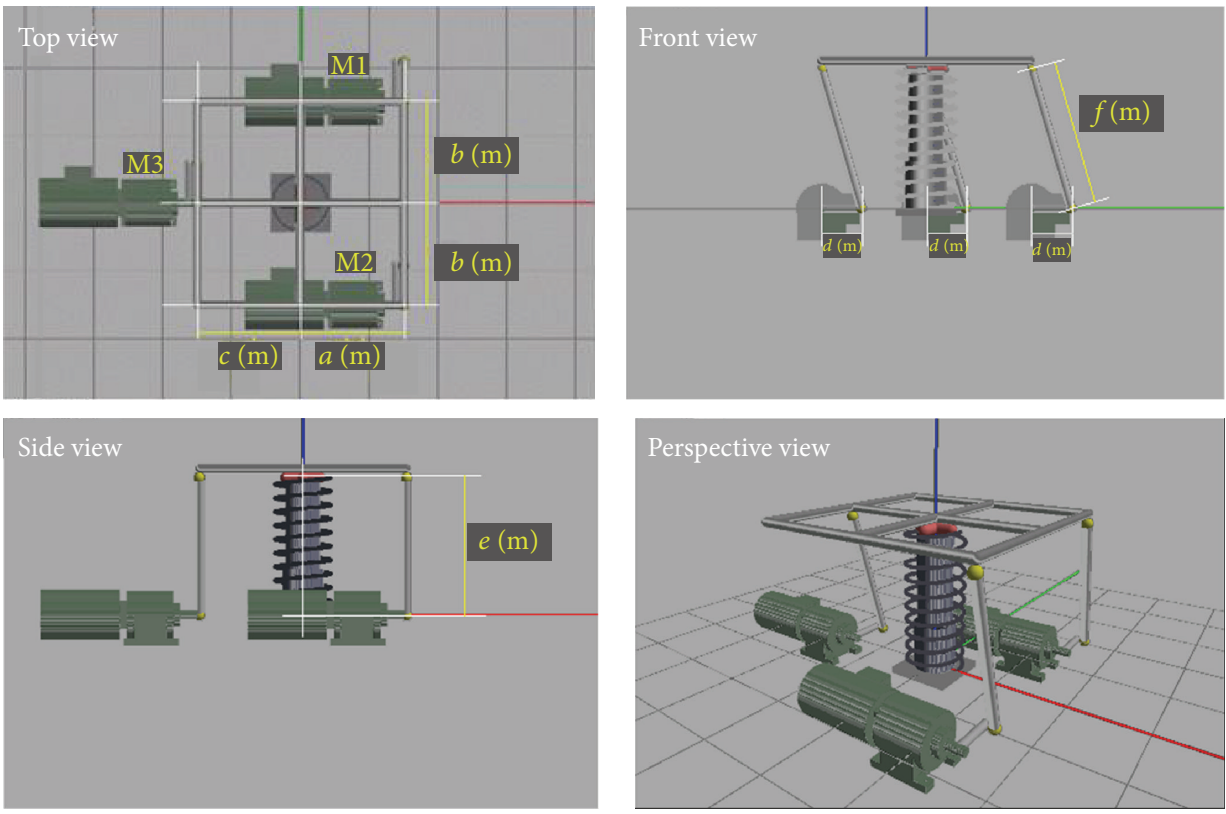

FIGURE 6: Simulation for 3DOF motion base dimension selection.

chairs, motion base, air injection, water spray, and vibration can be elements as well. In conventional studies, only personal experience was discussed for $4 \mathrm{D}$ element. In this paper, RS485 network topology, hundreds of 4D seats, and environment implementation were designed to be controlled using a repeater to RS485. Simulation results show that the improved digital cinema system is well designed for $4 \mathrm{D}$ chair and found to operate accurately under the condition of no traffic over the RS485.

\section{Conflict of Interests}

The authors declare that there is no conflict of interests regarding the publication of this paper.

\section{References}

[1] T. Yamaguchi, M. Nomura, K. Shirakawa, and T. Fujii, "SHD movie distribution system using image container with $4096 \times$ 
2160 pixel resolution and 36 bit color," in Proceedings of the IEEE International Symposium on Circuits and Systems (ISCAS '05), pp. 5918-5921, Kobe, Japan, May 2005.

[2] Digital Cinema Initiatives, Digital Cinema System Specification V1.1, 2007, http://www.dcimovies.com/.

[3] Specific Targeted Research Project, "IST call 2, Layered compression technologies for digital cinema to graphy and cross media conversion," WorldScreen, 2004, http://www.worldscreen.org.

[4] EDCine Project, "IST 6th framework program of the European Commission," https://ec.europa.eu/research/fp6/pdf/fp6-in-brief_en.pdf.

[5] WiMAX Forum, "Mobile WiMAX_Part I: A Technical Overview and Performance Evaluation," 2006, http://www.wimaxforum.org/.

[6] J. Leigh, L. Renambot, A. Johnson et al., "The global lambda visualization facility: an international ultra-high-definition wide-area visualization collaboratory," Future Generation Computer Systems, vol. 22, no. 8, pp. 964-971, 2006.

[7] T. Shimizu and D. Shirai, "International real-time streaming of $4 \mathrm{~K}$ digital cinema," Future Generation Computer Systems, vol. 22, no. 8, pp. 929-939, 2006.

[8] A. J. Woods, "How are crosstalk and ghosting defined in the stereoscopic literature?" in Stereoscopic Displays and Applications XXII, vol. 7863 of Proceedings of SPIE, San Francisco, Calif, USA, 2011.

[9] W.-P. K. Yiu, X. Jin, and S.-H. G. Chan, "Challenges and approaches in large-scale P2P media streaming," IEEE Multimedia, vol. 14, no. 2, pp. 50-59, 2007.

[10] H.-H. Yen, S. S. W. Lee, and B. Mukherjee, "Traffic grooming and delay constrained multicast routing in IP over WDM networks," in Proceedings of the IEEE International Conference on Communications (ICC '08), pp. 5246-5251, Beijing, China, May 2008.

[11] L. H. Sahasrabuddhe and B. Mukherjee, "Light-trees: optical multicasting for improved performance in wavelength-router networks," IEEE Communications Magazine, vol. 37, no. 2, pp. 67-73, 1999.

[12] T. Inoue and H. Ohzu, "Accommodative responses to stereoscopic three-dimensional display," Applied Optics, vol. 36, no. 19, pp. 4509-4515, 1997.

[13] C. Wheatstone, "Contributions to the physiology of visionpart the first. On some remarkable, and hitherto unobserved, phenomena of binocular vision," Philosophical Transactions of the Royal Society of London, vol. 128, pp. 371-394, 1838.

[14] N. A. Dodgson, A. J. Woods, J. O. Merritt, S. A. Benton, and M. T. Bolas, "Variation and extrema of human interpupillary distance," in Stereoscopic Displays and Virtual Reality Systems XI, vol. 5291 of Proceedings of SPIE, pp. 36-46, 2004.

[15] R. S. Allison, B. J. Gillam, and E. Vecellio, "Binocular depth discrimination and estimation beyond interaction space," Journal of Vision, vol. 9, no. 1, article 10, 2009.

[16] N. S. Holliman, J. P. Dakin, and R. G. W. Brown, Handbook of Optoelectronics, vol. 2, Taylor \& Francis, 2006.

[17] H.-J. Jia and Z.-H. Guo, "Research on the technology of RS485 over ethernet," in Proceedings of the International Conference on E-Product E-Service and E-Entertainment (ICEEE '10), pp. 1-3, November 2010. 


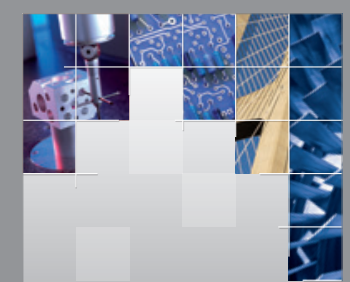

\section{Enfincering}
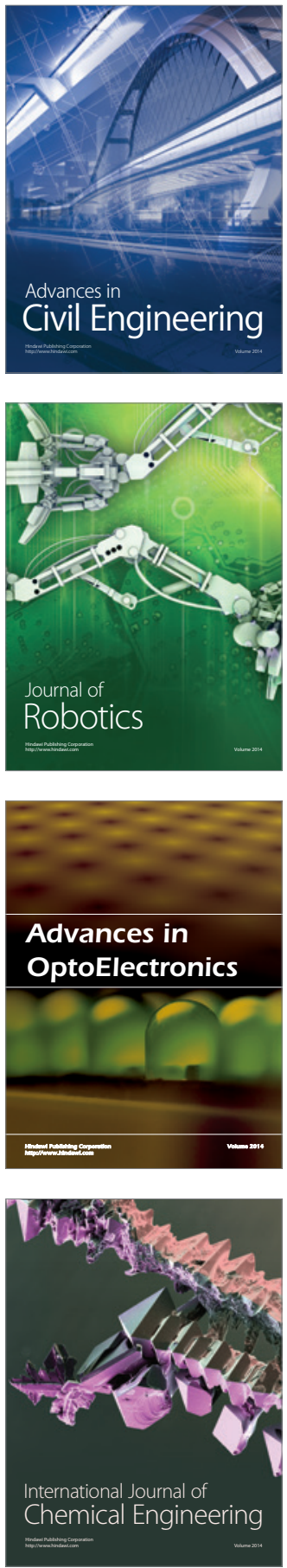

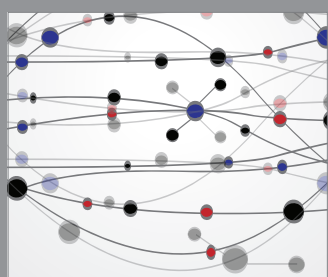

The Scientific World Journal

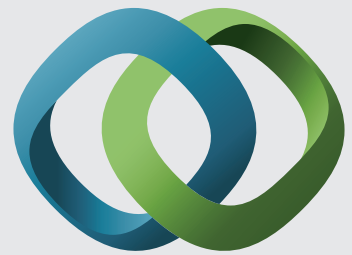

\section{Hindawi}

Submit your manuscripts at

http://www.hindawi.com
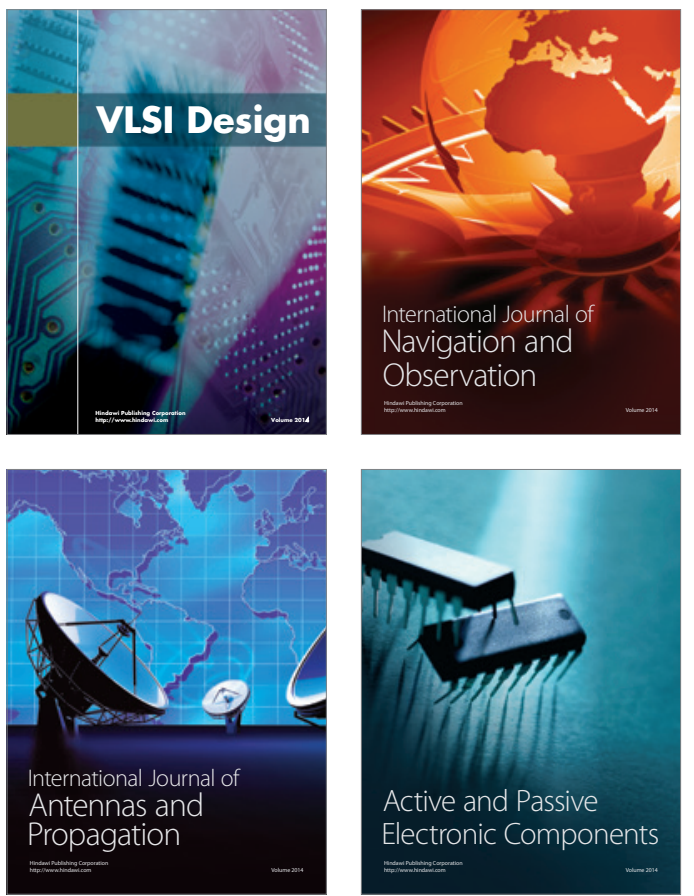
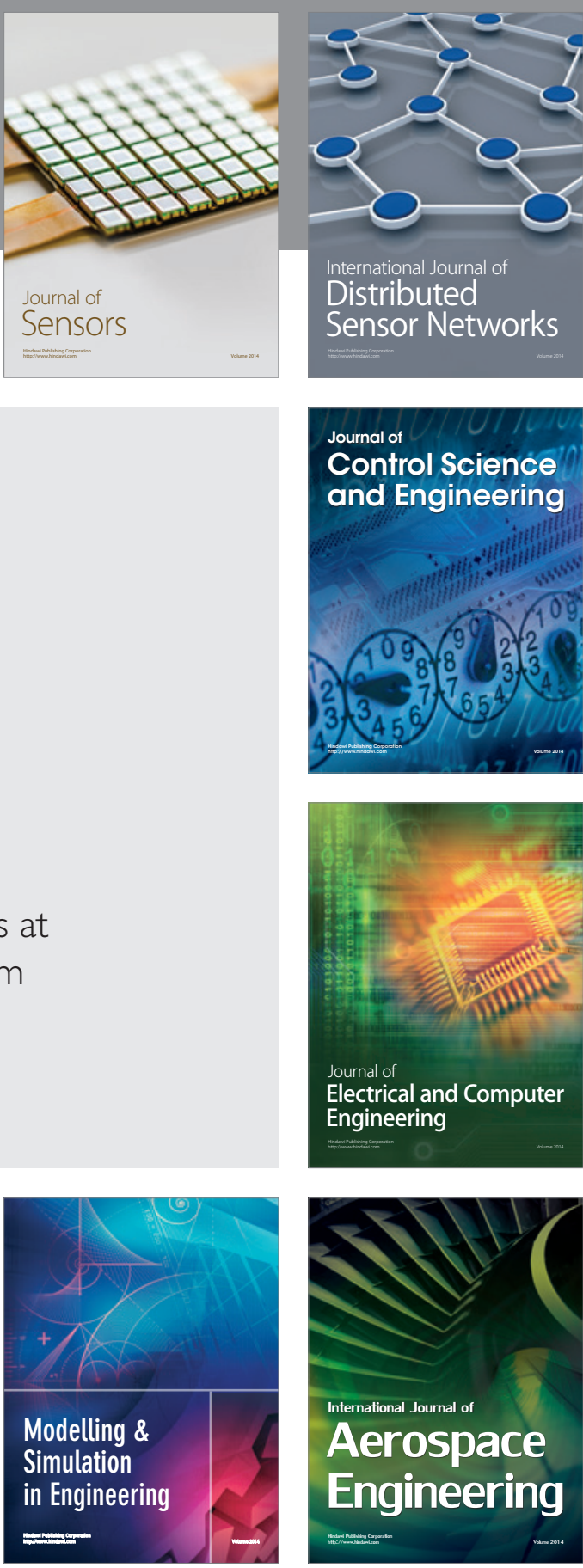

International Journal of

Distributed

Sensor Networks

Journal of

Control Science

and Engineering
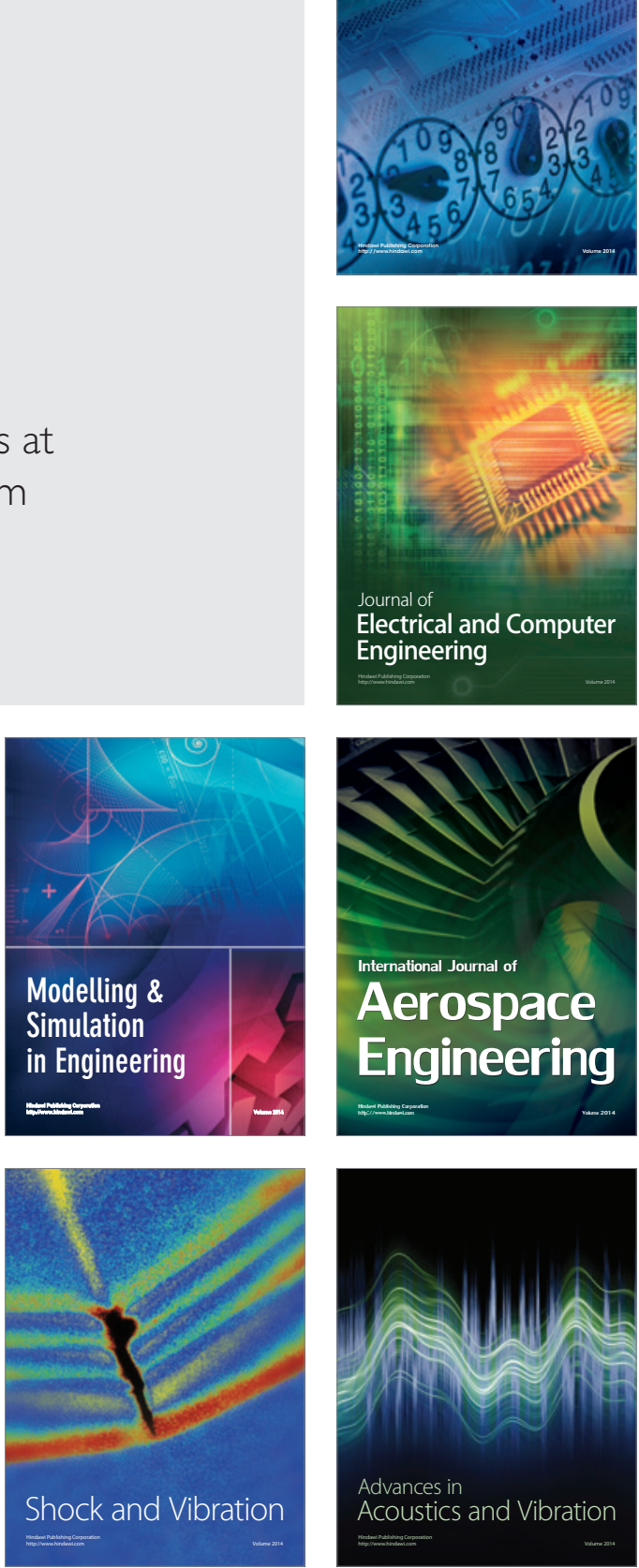